\title{
IRREDUCIBLE REPRESENTATIONS OF NORMAL SPACES
}

\author{
LEONARD R. RUBIN
}

(Communicated by James E. West)

\begin{abstract}
We define the notion of irreducible polyhedral representation of a normal space making use of approximate inverse systems. This generalizes the concept of irreducible polyhedral expansions introduced in 1937 by Freudenthal for metric compacta and generalized to uniform spaces by Isbell in 1961. We show that every normal space $X$ has an irreducible polyhedral representation whose dimension is $\operatorname{dim} X$ and whose weight is weight $(X)$. Approximate inverse systems were first introduced by $S$. Mardešic and this author. The concept generalizes that of inverse system and was essentially used in proving that each Hausdorff compactum of integral cohomological dimension $\leq n$ is the cell-like image of a Hausdorff compactum of covering dimension $\leq n$.
\end{abstract}

\section{INTRODUCTION}

In [M-R 1] we defined a new concept called approximate (inverse) system, which generalizes that of the classical (commutative) inverse system. We used the new notion to characterize covering dimension (dim) for compact Hausdorff spaces. Then in [M-R 2] we proved, relying heavily on the machinery of approximate systems, that if $X$ is a compact Hausdorff space whose cohomological dimension $\operatorname{dim}_{\mathbb{Z}} X \leq n$, then there is a compact Hausdorff space $Y$, $\operatorname{dim} Y \leq n$, and a cell-like map of $Y$ onto $X$.

Now we are going to explore the use of approximate systems in developing irreducible polyhedral representations of normal spaces in accordance with their dimension. This notion, to be defined below, is a type of generalization of irreducible polyhedral expansion first introduced for compact metric spaces in [Fr] and which was extended to the case of uniform spaces by J.R. Isbell [Is] and studied for compact Hausdorff spaces in [M1]. Since it is not generally possible to have a polyhedral expansion (in terms of finite polyhedra) for normal spaces, an entirely new concept is needed. This is why we shall speak of "representation" of a normal space, and as will be demonstrated, approximate systems are the perfect vehicles for such representations.

Received by the editors March 11, 1988 and, in revised form, April 16, 1989.

1980 Mathematics Subject Classification (1985 Revision). Primary 55M10, 54 S44.

Key words and phrases. Covering dimension, approximate inverse system, almost commutative system, irreducible representation. 
This work uses techniques developed in [M-R 1], [M-R 2]. In particular our Lemma 1 ( $\S 3$ ) is much like a lemma in [M-R 2] and our proof of the Theorem in this paper is developed along the lines of a proof in [M-R 1]. In fact, this research is an outgrowth of that done in [M-R 1], [M-R 2].

\section{APPROXIMATE SYSTEMS AND REPRESENTATIONS}

In this paper assume all spaces are Hausdorff and all polyhedra are compact, i.e., have finite triangulations. Dimension, dim, is defined in terms of finite open covers and finite open refinements ([En] or [Na]). By map we mean continuous function.

Definition 1. An approximate (inverse) system of metric compacta $\mathbf{X}=\left(X_{a}, \varepsilon_{a}\right.$, $\left.p_{a a^{\prime}}, A\right)$ consists of the following: A directed ordered set $(A, \leq)$ with no maximal element; for each $a \in A$, a compact metric space $X_{a}$ with metric $d=d_{a}$ and a real number $\varepsilon_{a}>0$; for each pair $a \leq a^{\prime}$ from $A$, a map $p_{a a^{\prime}}: X_{a^{\prime}} \rightarrow X_{a}$, satisfying the following conditions:

(A1) $d\left(p_{a_{1} a_{2}} p_{a_{2} a_{3}}, p_{a_{1} a_{3}}\right) \leq \varepsilon_{a_{1}}, a_{1} \leq a_{2} \leq a_{3} ; p_{a a}=i d$.

(A2) $(\forall a \in A)(\forall \eta>0)\left(\exists a^{\prime} \geq a\right)\left(\forall a_{2} \geq a_{1} \geq a^{\prime}\right)$ $d\left(p_{a a_{1}} p_{a_{1} a_{2}}, p_{a a_{2}}\right) \leq \eta$.

(A3) $(\forall a \in A)(\forall \eta>0)\left(\exists a^{\prime} \geq a\right)\left(\forall a^{\prime \prime} \geq a^{\prime}\right)\left(\forall x, x^{\prime} \in X_{a^{\prime \prime}}\right)$ $d\left(x, x^{\prime}\right) \leq \varepsilon_{a^{\prime \prime}} \Rightarrow d\left(p_{a a^{\prime \prime}}(x), p_{a a^{\prime \prime}}\left(x^{\prime}\right)\right) \leq \eta$.

We refer to the numbers $\varepsilon_{a}$ as the meshes of $\mathbf{X}$.

If $\pi_{a}: \prod_{a \in A} X_{a} \rightarrow X_{a}, a \in A$, denote projections, we define the limit space $X=\lim \mathbf{X}$ and the natural projections $p_{a}: X \rightarrow X_{a}$ as follows.

Definition 2. A point $x=\left(x_{a}\right) \in \Pi X_{a}$ belongs to $X=\lim \mathbf{X}$ provided for every $a \in A$,

$$
x_{a}=\lim _{a_{1}} p_{a a_{1}}\left(x_{a_{1}}\right) .
$$

The natural projection $p_{a}=\pi_{a} \mid X: X \rightarrow X_{a}$.

Definition 3. We say $\left\{X_{a}, p_{a a^{\prime}}, A\right\}$ is an almost commutative system [M-S] if there exist numbers $\varepsilon_{a}>0, a \in A$, so that $\left\{X_{a}, \varepsilon_{a}, p_{a a^{\prime}}, A\right\}$ is an approximate system.

Definition 4. Let $K$ be a complex and let $f, g: X \rightarrow|K|$ be maps. We say that $g$ is a $K$-modification of $f$ if for every $x \in X$ and $\sigma \in K, f(x) \in \sigma$ implies $g(x) \in \sigma$. Note that a simplicial approximation $\varphi: K_{1} \rightarrow K_{2}$ of a map $\pi:\left|K_{1}\right| \rightarrow\left|K_{2}\right|$ is a $K_{2}$-modification of $\pi$. Moreover, if $K^{\prime}$ is a subdivision of $K$, and $g: X \rightarrow\left|K^{\prime}\right|$ is a $K^{\prime}$-modification of $f: X \rightarrow\left|K^{\prime}\right|=|K|$, then $g$ is also a $K$-modification of $f$.

Definition 5. A map $f: X \rightarrow|K|$ is $K$-irreducible if for every $K$-modification $g$ of $f$, one has $g(X)=|K|$. Since $f$ is its own $K$-modification, a $K$ irreducible map $f$ is onto. A map $f: X \rightarrow P$ where $P$ is a polyhedron is 
called irreducible if it is $K$-irreducible for some triangulation $K$ of $P$. Note that every irreducible map $f: X \rightarrow P$ is onto.

We now come to our definition of irreducible polyhedral representation.

Definition 6. Let $X$ be a normal space and $\mathbf{P}=\left\{P_{a}, p_{a a^{\prime}}, A\right\}$ be an almost commutative system of compacta $P_{a}$. A set of maps $f_{a}: X \rightarrow P_{a}, a \in A$, is said to be a representation of $X$ in $\mathbf{P}$ if the map $f: X \rightarrow \prod_{a \in A} P_{a}$ given by $f(x)=\left(f_{a}(x)\right)$ embeds $X$ onto a dense subspace of $\lim \mathbf{P}$. We say the representation is of dimension $\leq n$ if $\operatorname{dim} P_{a} \leq n$ for all $a$, and we define its weight to be card $(A)$. We call it cofinite if the indexing set $A$ is cofinite, i.e., for each $a \in A$ there are only finitely many $a^{\prime} \in A$ with $a^{\prime} \leq a$.

We call the representation polyhedral if each $P_{a}$ is a polyhedron and irreducible if in addition each $f_{a}: X \rightarrow P_{a}$ is irreducible and for $a \leq a^{\prime}, p_{a a^{\prime}}$ is irreducible. A polyhedral representation will be called simplicially irreducible if also for each $a$ there is a fixed triangulation $K_{a}$ of $P_{a}$ so that $f_{a}$ is $K_{a}$ irreducible and whenever $a \leq a^{\prime}$, then $p_{a a^{\prime}}$ carries $K_{a^{\prime}}$ simplicially to a subdivision of $K_{a}$ and $p_{a a^{\prime}} \circ f_{a^{\prime}}$ is a $K_{a}$-modification of $f_{a}: X \rightarrow P_{a}$.

Note. If $f_{a}$ is $K_{a}$-irreducible, then the latter condition is readily seen to imply that $p_{a a^{\prime}}$ is $K_{a}$-irreducible also. Hence simplicially irreducible implies irreducible.

Our main theorem is the following.

Theorem. Every normal space $X$ has a cofinite simplicially irreducible representation of dimension $\leq \operatorname{dim} X$, and of weight $\leq$ weight $(X)$.

We delay proof until $\S 4$. The following Corollary appears as Corollary 3 in [M1] and elsewhere.

Corollary. If $X$ is a normal space and $\operatorname{dim} X \leq n$, then $X$ has a Hausdorff compactification $P$ such that $\operatorname{dim} P \leq n$.

Proof. Let $P=\lim \mathbf{P}$ and apply Theorems 2 and 4 of [M-R 1].

\section{BASIC LEMMA}

The next lemma is basic to the later construction. It is similar to Lemma 2 of [M1] and Lemma 1 of [M-R 2].

Lemma 1. Let $X$ be a normal space, let $f_{i}: X \rightarrow P_{i}=\left|K_{i}\right|$ be maps to triangulated polyhedra, and let $\varepsilon_{i}>0, i=1, \cdots, k$. Then there exist a polyhedron $Q=|L|, \operatorname{dim} Q \leq \operatorname{dim} X$, an L-irreducible map $g: X \rightarrow Q$, and maps $p_{i}: Q \rightarrow P_{i}$ which are simplicial from $L$ to some subdivision $L_{i}$ of $K_{i}$ such that $d\left(f_{i}, p_{i} g\right) \leq \varepsilon_{i}$ and $p_{i} g$ is an $L_{i}$-modification of $f_{i}, i=1, \cdots, k$. The subdivision $L_{i}$ may be given arbitrarily fine mesh. Moreover, if for a given index $i$ the map $f_{i}$ is $K_{i}$-irreducible, then the corresponding map $p_{i}$ is $L_{i}$-irreducible. Proof. Let $L_{i}$ be a subdivision of $K_{i}$ with

$$
\text { mesh } L_{i} \leq \varepsilon_{i} / 2 \text {. }
$$


Note that $f_{i}$ is $L_{i}$-irreducible if it is $K_{i}$-irreducible. Let $P=P_{1} \times \cdots \times P_{k}$, let $f=f_{1} \times \cdots \times f_{k}: X \rightarrow P$, and let $\pi_{i}: P \rightarrow P_{i}, i=1, \cdots, k$, be the projections. Choose $\delta>0$ so small that

$$
d\left(x, x^{\prime}\right) \leq \delta \Rightarrow d\left(\pi_{i}(x), \pi_{i}\left(x^{\prime}\right)\right) \leq \varepsilon_{i} / 2, i=1, \cdots, k .
$$

Let $K$ be a triangulation of $P$ so fine that

$$
\text { mesh } K \leq \delta \text {, }
$$

and the projections $\pi_{i}:|K| \rightarrow\left|L_{i}\right|$ admit simplicial approximations $\bar{p}_{i}: K \rightarrow$ $L_{i}, i=1, \cdots, k$. Since $\bar{p}_{i}$ is an $L_{i}$-modification of $\pi_{i}$, we have

$$
d\left(\bar{p}_{i}, \pi_{i}\right) \leq \operatorname{mesh} L_{i} \leq \varepsilon_{i} / 2 .
$$

Let $\mathscr{U}$ be the (finite) open cover of $X$ consisting of the sets $f^{-1}(s t(v)), v$ a vertex of $K$. Choose a finite open cover $\mathscr{V}$ of $X$ so that $\mathscr{V}$ refines $\mathscr{U}$ and so that $\operatorname{dim}(\mathscr{N}) \leq \operatorname{dim} X$, where $\mathscr{N}$ is the nerve of $\mathscr{V}$. Let $g_{0}: X \rightarrow|\mathcal{N}|$ be a canonical map.

There is a simplicial map $h_{0}: \mathcal{N} \rightarrow K$ defined on vertices of $\mathscr{N}$ by sending $V \in \mathscr{V}$ to a vertex $v$ of $K$ such that $V \subset f^{-1}(s t(v))$. There is a subcomplex $L$ of $\mathscr{N}$ and an $\mathscr{N}$-modification $g: X \rightarrow|L|$ of $g_{0}$ such that $g$ is $L$-irreducible. This follows by choosing a minimal subcomplex $L$ of $\mathscr{N}$ for which there is an $\mathscr{N}$-modification $g$ into $|L|$ and then applying Lemma 2 below.

Put $Q=|L|$ and note that $\operatorname{dim} Q \leq \operatorname{dim}|\mathcal{N}| \leq \operatorname{dim} X$. Let $h=h_{0} \mid Q: Q \rightarrow$ $|K|$; we see that $h: L \rightarrow K$ is simplicial. Choose $p_{i}$ to be $\bar{p}_{i} h: Q \rightarrow\left|L_{i}\right|=P_{i}$. Then $p_{i}: L \rightarrow L_{i}$ is simplicial.

Note that $d(f, h g) \leq$ mesh $K \leq \delta$, and therefore,

$$
d\left(\pi_{i} f, \pi_{i} h g\right)=d\left(f_{i}, \pi_{i} h g\right) \leq \varepsilon_{i} / 2, i=1, \cdots, k .
$$

From (4) we get $d\left(\bar{p}_{i} h, \pi_{i} h\right) \leq \varepsilon_{i} / 2$. But $\bar{p}_{i} h=p_{i}$, so we have,

$$
d\left(p_{i}, \pi_{i} h\right) \leq \varepsilon_{i} / 2 \text {. }
$$

Hence,

$$
d\left(p_{i} g, \pi_{i} h g\right) \leq \varepsilon_{i} / 2
$$

Now (5) and (7) yield

$$
d\left(f_{i}, p_{i} g\right) \leq \varepsilon_{i} .
$$

Next we will show that if $f_{i}$ is $K_{i}$-irreducible, hence $L_{i}$-irreducible, then $p_{i}$ is $L_{i}$-irreducible. If we can show that $p_{i} g$ is $L_{i}$-irreducible, then certainly $p_{i}$ will be $L_{i}$-irreducible. It is sufficient to show that $p_{i} g$ is an $L_{i}$-modification of $f_{i}$.

Choose $x \in X$ and suppose $f_{i}(x)=\pi_{i} f(x)$ lies in the interior of the simplex $\sigma$ of $L_{i}$. The choices of $g$ and $h$ are such that if for a simplex $\tau$ of $K, f(x)$ lies in $\dot{\tau}$, then $h g(x) \in \tau$. Since $\bar{p}_{i}$ is a simplicial approximation of $\pi_{i}$, then $\bar{p}_{i}(f(x)) \in \sigma$ and hence $\bar{p}_{i}(\tau) \subset \sigma$. Thus $\bar{p}_{i} f(x)$ and $\bar{p}_{i} h g(x)=p_{i} g(x)$ lie 
in $\sigma$. This shows that both $f_{i}(x)$ and $p_{i} g(x)$ lie in $\sigma$, so $p_{i} g$ is an $L_{i}$ modification of $f_{i}$ and the proof is complete.

A map $g: X \rightarrow|L|$ is said to be essential on a simplex $\sigma$ of $L$ if there is no map $f: g^{-1}(\sigma) \rightarrow \partial \sigma$ such that $f$ agrees with $g$ on $g^{-1}(\partial \sigma)$.

Lemma 2. Let $X$ be a normal space, $L$ a finite complex, and $g: X \rightarrow|L|$ be a map such that no L-modification of $g$ carries $X$ into a proper subcomplex of $L$. Then $g$ is L-irreducible.

Proof. It is sufficient to show that $g$ is surjective; for every $L$-modification of $g$ satisfies the hypotheses of this lemma.

Suppose $\lambda$ is a principal simplex of $L$. Then $g$ must be essential on $\lambda$ or else there would be an $L$-modification of $g$ whose image contained no points of the interior of $\lambda$. But as argued in the proof of 3.6 of [R-S], this implies that $g$ is essential on every simplex of $L$ and hence $g$ maps onto the interior of every simplex of $L$. This proves that $g$ is surjective.

Note. Lemma 2 is still true for infinite complexes for which each simplex lies in a principal simplex, e.g., locally finite dimensional complexes.

\section{Proof of THEOREM}

The reader will notice many similarities between the forthcoming proof and the proof of Theorem 5 of [M-R 1]. We shall avoid repetition where possible, but some redundancy is necessary to maintain the integrity of the current proof.

As in [M-R 1], choose an embedding $e$ of $X$ into a Tihonov cube $Y=I^{\tau}$ where $\tau=$ weight $(X)$ and take $Y$ to be the inverse limit of an (commutative) inverse system $\left(Y_{a}, q_{a a^{\prime}}, A\right)$ of finite dimensional cubes where $A$ is cofinite with no maximal element and card $(A) \leq$ weight $(X)$. For simplicity, assume $X \subset$ $Y$, i.e., that $e$ is the inclusion map. Let $q_{a}: Y \rightarrow Y_{a}$ be the natural projections and let $|a| \geq 0$ denote the number of predecessors of $a \in A$. Using Lemma 1 of the current paper instead of Lemma 5 of [M-R 1], obtain the following data: for each $a \in A$, a compact triangulated polyhedron $P_{a}=\left|K_{a}\right|, \operatorname{dim} P_{a} \leq \operatorname{dim} X$, maps $f_{a}: X \rightarrow P_{a}, h_{a}: P_{a} \rightarrow Y_{a}$ and numbers $\varepsilon_{a}>0, \delta_{a}>0$ and for each pair $a \leq a^{\prime}$ a map $p_{a a^{\prime}}: P_{a^{\prime}} \rightarrow P_{a}$. We require that each $f_{a}: X \rightarrow P_{a}$ be $K_{a}$-irreducible and that the following conditions hold (see 5.1 of [M-R 1]).

1. $d\left(p_{a a^{\prime}} f_{a^{\prime}}, f_{a}\right) \leq \frac{\varepsilon_{a}}{3\left|a^{\prime}\right|-|a|}, a<a^{\prime}, p_{a a}=i d$.

2. $d\left(q_{a} \mid X, h_{a} f_{a}\right) \leq \delta_{a} / 3$,

3. $x, x^{\prime} \in P_{a}, d\left(x, x^{\prime}\right) \leq \varepsilon_{a} \Rightarrow d\left(h_{a}(x), h_{a}\left(x^{\prime}\right) \leq \delta_{a} / 3\right.$,

4. $x, x^{\prime} \in P_{a^{\prime}}, d\left(x, x^{\prime}\right) \leq \varepsilon_{a^{\prime}} \Rightarrow d\left(p_{a a^{\prime}}(x), p_{a a^{\prime}}\left(x^{\prime}\right)\right) \leq \frac{\varepsilon_{a}}{3\left|a^{\prime}\right|-|a|}$,

5. $y, y^{\prime} \in Y_{a}, d\left(y, y^{\prime}\right) \leq \delta_{a^{\prime}} \Rightarrow d\left(q_{a a^{\prime}}(y), q_{a a^{\prime}}\left(y^{\prime}\right)\right) \leq \frac{\delta_{a}}{3\left|a^{\prime}\right|-|a|}$,

6. $p_{a a^{\prime}}$ carries $K_{a^{\prime}}$ simplicially to a subdivision $L_{a}^{a^{\prime}}$ of $K_{a}, p_{a a^{\prime}} f_{a^{\prime}}$ is an $L_{a}^{a^{\prime}}$-modification of $f_{a}$, and mesh $\left(L_{a}^{a^{\prime}}\right) \leq \frac{1}{\left|a^{\prime}\right|+1}, a \leq a^{\prime}$.

The verification that $\mathbf{P}=\left(P_{a}, \varepsilon_{a}, p_{a a^{\prime}}, A\right)$ is an approximate system is precisely as in 5.2 of [M-R 1]. Consider the map $f: X \rightarrow \prod_{a \in A} P_{a}$ given by 
$f(x)=\left(f_{a}(x)\right)$. We claim that each $f(x)$ is a thread of $\mathbf{P}$. It is necessary to show that for a given $a \in A$,

7. $f_{a}(x)=\lim _{a^{\prime}} p_{a a^{\prime}} f_{a^{\prime}}(x)$.

However, it is clear that $\lim _{a^{\prime} \geq a}\left|a^{\prime}\right|=\infty$. Therefore an application of 6 yields 7 .

The injectivity of $f$ goes as in 5.4 of [M-R 1] and that $f$ carries $X$ onto a dense subspace of $\lim \mathbf{P}$ is obtained from the proof of 5.5 therein (note though, that you should set $f_{a}=g_{a}$ ). To show that $f$ is an embedding, we shall show that $f$ is a closed map of $X$ to $f(X)$.

Let $B$ be closed in $X$ and suppose $x \in X \backslash B$. We shall find an $a^{\prime} \in A$ such that

8. $d\left(f_{a^{\prime}}(b), f_{a^{\prime}}(x)\right)>\varepsilon_{a^{\prime}}$ for all $b \in B$.

Hence with $U$ as the $\varepsilon_{a^{\prime}}$-neighborhood of $f_{a^{\prime}}(x)$ in $P_{a^{\prime}}$, we have $U \cap$ $f_{a^{\prime}}(B)=\phi$. Then by Lemma 3 of [M-R 1], $p_{a^{\prime}}^{-1}(U)$ is a neighborhood of $f(x)$ in $\lim \mathbf{P}$ which does not intersect $f(B)$.

Note that $x \notin \bar{B}$ (closure in $Y$ ). Hence there exists $a \in A$ and $\delta>0$ such that $d\left(q_{a}(\bar{B}), q_{a}(x)\right)>\delta$. Thus $d\left(q_{a}(B), q_{a}(x)\right)>\delta$. Choose $k$ so large that $\frac{\delta_{a}}{3^{k-|a|}}<\delta$. Select $a^{\prime} \in A$ so that $\left|a^{\prime}\right| \geq k$ and $a^{\prime} \geq a$. Then 5 and the fact that $\left(Y_{a}, q_{a a^{\prime}}, A\right)$ is a commutative system show us that

9. $d\left(q_{a^{\prime}}(b), q_{a^{\prime}}(x)\right)>\delta_{a^{\prime}}$ for all $b \in B$.

Suppose it were true that $d\left(f_{a^{\prime}}(b), f_{a^{\prime}}(x)\right) \leq \varepsilon_{a^{\prime}}$ for some $b \in B$. This and 3 would imply,

10. $d\left(h_{a^{\prime}} f_{a^{\prime}}(b), h_{a^{\prime}} f_{a^{\prime}}(x)\right) \leq \delta_{a^{\prime}} / 3$.

But 2 yields,

11. $d\left(q_{a^{\prime}}(x), h_{a^{\prime}} f_{a^{\prime}}(x)\right) \leq \delta_{a^{\prime}} / 3$ and

12. $d\left(q_{a^{\prime}}(b), h_{a^{\prime}} f_{a^{\prime}}(b)\right) \leq \delta_{a^{\prime}} / 3$.

Using $12,10,11$, we would get, $d\left(q_{a^{\prime}}(b), q_{a^{\prime}}(x)\right) \leq \delta_{a^{\prime}}$, which contradicts 9 . Hence we conclude,

13. $d\left(f_{a^{\prime}}(b), f_{a^{\prime}}(x)\right)>\varepsilon_{a^{\prime}}$ for all $b \in B$.

The proof is now complete.

\section{REFERENCES}

[En] R. Engelking, Dimension Theory, PWN-Polish Scientific Publishers, Warsaw, 1978.

[Fr] H. Freudenthal, Entwicklungen von Räumen und ihren Gruppen, Compositio Math. 4 (1937), 145-234.

[Hu] S. T. Hu, Theory of Retracts, Wayne State University Press, Detroit, Michigan, 1965.

[Is] J. R. Isbell, Irreducible polyhedral expansions, Indag. Math. 23 (1961), 242-248.

[M1] S. Mardešić, On covering dimension and inverse limits of compact spaces, Illinois J. Math. 4 (1960), 278-291.

[M-R 1] S. Mardešić and L. Rubin, Approximate inverse systems of compacta and covering dimension, Pacific J. Math. 138 (1989), 129-144. 
[M-R 2] _ Cell-like mappings and non-metrizable compacta of finite cohomological dimension, Trans. Amer. Math. Soc. (to appear).

[M-S] S. Mardešić and J. Segal, Stability of almost commutative inverse systems of compacta, Topology Appl. 31 (1989), 285-299.

[Na] J. Nagata, Modern dimension theory, Heldermann Verlag, Berlin, 1983.

[R-S] L. Rubin and P. Schapiro, Cell-like maps onto non-compact spaces of finite cohomological dimension, Topology Appl. 27 (1987), 221-244.

Department of Mathematics, University of Oklahoma, 601 Elm Ave., Rm. 423, Norman, OKLAHOMA 73019 